\title{
Some bounds on the generalised total chromatic number of degenerate graphs
}

\author{
Izak Broere \\ Department of Mathematics and Applied Mathematics \\ University of Pretoria, Pretoria, South Africa \\ Gabriel Semanišin \\ Institute of Computer Science, \\ Faculty of Science, P.J. Šafárik University, Košice, Slovakia
}

\section{Abstract}

The total generalised colourings considered in this paper are colourings of the vertices and of the edges of graphs satisfying the following conditions:

- each set of vertices of the graph which receive the same colour induces an $m$-degenerate graph,

- each set of edges of the graph which receive the same colour induces an $n$-degenerate graph, and

- incident elements receive different colours.

Bounds for the least number of colours with which this can be done for all $k$-degenerate graphs are obtained.

Keywords: total colouring number, graph property, $k$-degenerate graph 2010 MSC: 05C15, 05C70

Email addresses: izak.broere@up.ac.za (Izak Broere), gabriel.semanisin@upjs.sk (Gabriel Semanišin) 


\section{Introduction}

For graphs in general, we use the notation and terminology of [4]; for concepts related to (hereditary) graph properties we use the notation and terminology of [1]. Two particular graph properties to be used in the sequel are $\mathcal{O}$ and $\mathcal{O}_{1}$, where $\mathcal{O}=\{G \in \mathcal{I}: G$ is edgeless, i.e., $E(G)=\emptyset\}$ and $\mathcal{O}_{k}=\{G \in \mathcal{I}$ : each component of $G$ has at most $k+1$ vertices $\}$ and $\mathcal{I}$ is the set of all graphs.

A graph $G$ is called $k$-degenerate if the minimum degree $\delta(H) \leq k$ for each induced subgraph $H$ of $G$. The set of all $k$-degenerate graphs will be denoted by $\mathcal{D}_{k}$; it is a well-known additive induced hereditary graph property. $k$-degenerate graphs were introduced in [8] and they play an important role in the structure of hereditary properties of graphs (see e.g. [9], [10]).

Let $\mathcal{P}$ and $\mathcal{Q}$ be graph properties and let $C=\{1, \ldots, d\}$. If $G=(V, E)$ is a graph, then a function $c: V \cup E \rightarrow C$ is a total $(\mathcal{P}, \mathcal{Q})$-colouring of $G$ in $d$ colours if

$$
G\left[\left\{c^{-1}(i)\right\} \cap V\right] \in \mathcal{P} \text {, for all } i \in C,
$$

(2) $G\left[\left\{c^{-1}(i)\right\} \cap E\right] \in \mathcal{Q}$, for all $i \in C$,

(3) if $e=v u \in E$ (with $v, u \in V)$, then $c(v) \neq c(e)$ and $c(u) \neq c(e)$, i.e., no vertex receives the same colour as any edge incident to it.

The minimum number of colours needed in a total $(\mathcal{P}, \mathcal{Q})$-colouring of $G$ is

called the total $(\mathcal{P}, \mathcal{Q})$-chromatic number and is denoted by $\chi_{\mathcal{P}, \mathcal{Q}}^{\prime \prime}(G)$ (see $[2])$. Clearly, when $\mathcal{P}=\mathcal{O}$ and $\mathcal{Q}=\mathcal{O}_{1}$, a total $(\mathcal{P}, \mathcal{Q})$-colouring of a graph

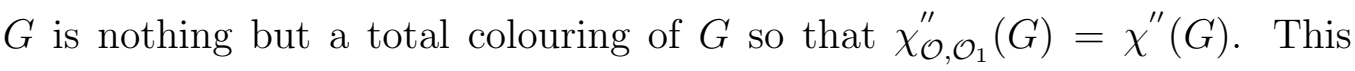


parameter is studied in [7] where it is shown that an $s$-degenerate graph has a total colouring with $\Delta+1$ colours if the maximum degree $\Delta$ is sufficiently large.

\section{Motivation}

To know the minimum number, or at least a bound for the minimum number of colours needed in a total $(\mathcal{P}, \mathcal{Q})$-colouring of a graph $G$, implies that we know in how many parts we can partition the vertices and the edges of the graph separately while imposing a restriction on the structure of each of these parts. In fact, we impose restrictions on the subgraph induced by each vertex part (by choosing a suitable $\mathcal{P}$ ) which are independent of the restrictions posed on the subgraph induced by each edge part (by choosing a suitable $\mathcal{Q}$ ). We shall now describe a possible application of this type of partition problem for networks which can be represented as graphs.

The theory of wireless sensor networks has become important in our modern day and age - see [3] for example. This is due to its many potential applications in process management, health care, environmental sensing, etc. Furthermore, this theory has interesting challenging theoretical problems.

A wireless sensor network (WSN) differs from a computer network in that it has limited capabilities of the sensors which could be caused by low energy sources or low computational capacity. Pairs of sensors typically communicate through designated channels. Because of potential collisions and interferences and a limited capability of the sensor involved, the number of communication channels linked to one sensor may be limited. In order to secure the communication sent through this network one can assign certificates 
to sensors. Again, due to limited computational capacity, it is only possible to use a fixed, but limited, number of certificates. On the other hand, the communication will be safer, if the same certificate is not used repeatedly. Such a network may therefore fail to function far away from maintenance engineers, deep under the sea or in outer space for example, if the limitation specifications imposed in its design are not strict enough for it to handle its task. It is therefore a reasonable option to structure its design in such a way that some parts of the WSN may still function optimally in such a situation. This option calls for labellings of the sensors and the communication channels during the design phase of the WSN in such a way that the subnetworks determined by sets of equally labelled sensors and equally labelled communication channels have suitable structural limitations to make parts of the network still functional.

This situation corresponds to a great extent to the problem we study in this paper: Think about the network as the graph $G$ having as vertex set $V$ the set of sensors and as edge set $E$ the set of its communication channels. The limited number of communication channels linking one sensor to others may then be translated into a degree restriction for the vertices of the graph linking it to the graph $G$ being $k$-degenerate for a suitable choice of $k$.

By determining for such a graph $G$ its total $\left(\mathcal{D}_{m}, \mathcal{D}_{n}\right)$-chromatic number $\chi_{\mathcal{D}_{m}, \mathcal{D}_{n}}^{\prime \prime}(G)$, one obtains information on how many subnetworks of a similar kind, which could ensure that such subnetworks remain functional in case of a failure of the WSN, are needed. Condition (3) in the definition of a total $(\mathcal{P}, \mathcal{Q})$-colouring of a graph is perhaps not applicable to this situation. However, any upper bound on the number of colours needed can only be 
improved on by relaxing this condition.

Our particular choice of degree restrictions of the vertices of the subnetworks ensure stricter restrictions on its structural design. It was shown in [6] that WSN with degenerate topologies possesses specific properties that are very important for communication protocol design.

In this paper we then study, for positive integers $m, n$ and $k$, the total $\left(\mathcal{D}_{m}, \mathcal{D}_{n}\right)$-chromatic number $\chi_{\mathcal{D}_{m}, \mathcal{D}_{n}}^{\prime \prime}(G)$ of a graph $G$ with $G \in \mathcal{D}_{k}$.

\section{The total colouring of degenerate graphs}

In our first result we give an upper bound for $\chi_{\mathcal{D}_{m}, \mathcal{D}_{n}}^{\prime \prime}(G)$ for a graph $G \in \mathcal{D}_{k}$.

Theorem 1. For every three positive integers $m, n$ and $k$ and for every $G \in$ $\mathcal{D}_{k}$ we have $\chi_{\mathcal{D}_{m}, \mathcal{D}_{n}}^{\prime \prime}(G) \leq \max \left\{\left\lceil\frac{k+1}{m+1}\right\rceil,\left\lceil\frac{k}{n}\right\rceil+2\right\}$.

Proof. Consider any three positive integers $m, n$ and $k$. We denote, for convenience, the number $\max \left\{\left\lceil\frac{k+1}{m+1}\right\rceil,\left\lceil\frac{k}{n}\right\rceil+2\right\}$ by $x$. The proof is by induction over the number of vertices of $G$. If $G$ has only one vertex, the result holds since then $\chi_{\mathcal{D}_{m}, \mathcal{D}_{n}}^{\prime \prime}(G)=1$ while $x \geq 3$ for all positive integers $m, n$ and $k$.

Hence suppose the result holds for all $k$-degenerate graphs of order at most $p-1$ and let $G$ be one of order $p$. Then $G$ has a vertex of degree at most $k$; suppose $v$ is such a vertex. Since $G-v$ is also $k$-degenerate,

the induction hypothesis assures us that $\chi_{\mathcal{D}_{m}, \mathcal{D}_{n}}^{\prime \prime}(G-v) \leq x$. Consider a total $\left(\mathcal{D}_{m}, \mathcal{D}_{n}\right)$ colouring of $G-v$ using $x$ colours, which we will denote by $1,2, \ldots, x$, and let, $W_{1}, W_{2}, \ldots, W_{x}$ be the colour classes into which the 
subset of $V(G-v)$ consisting of those vertices which are adjacent to $v$ is partitioned by this colouring of the vertices of $G-v$.

We claim that at least one set $W_{i}$ then contains at most $m$ vertices. This is so since $x \geq\left\lceil\frac{k+1}{m+1}\right\rceil$ and hence $x \geq \frac{k+1}{m+1}$, i.e., $x(m+1) \geq k+1$. Hence, if each $W_{i}$ contains at least $m+1$ vertices, then the degree of $v$ is $\left|\bigcup_{j} W_{j}\right| \geq x(m+1) \geq k+1$ which contradicts the fact that the degree of $v$ is at most $k$.

Therefore at least one of the $W_{i}$ 's, say $W_{x}$, contains at most $m$ vertices: we can therefore colour $v$ with $x$ to complete the colouring of the vertices of $G$ with $x$ colours such that each colour class of vertices induces an $m$-degenerate graph as required.

In order to colour the edges incident to $v$ without violating the incidence condition, each of the $k$ edges incident to $v$ must be coloured by a colour different from the colours of its endvertices; we shall call such a colour admissible at the edge. This means that we have $x-2$ possibilities for each edge incident to a vertex with colour different from $x$ and $x-1$ possibilities for each edge of which both endvertices are coloured by $x$.

We shall show that we can assign colours to the edges incident to $v$ is such a way that:

- the incidence condition for vertices and edges will not be violated;

- any colour will be used for at most $n$ edges incident to $v$.

Clearly, once this is done the induction step is completed and we have a total colouring of $G$ with the desired properties.

In order to show it, let us construct a network $N$ corresponding to $G$ in 
the following way:

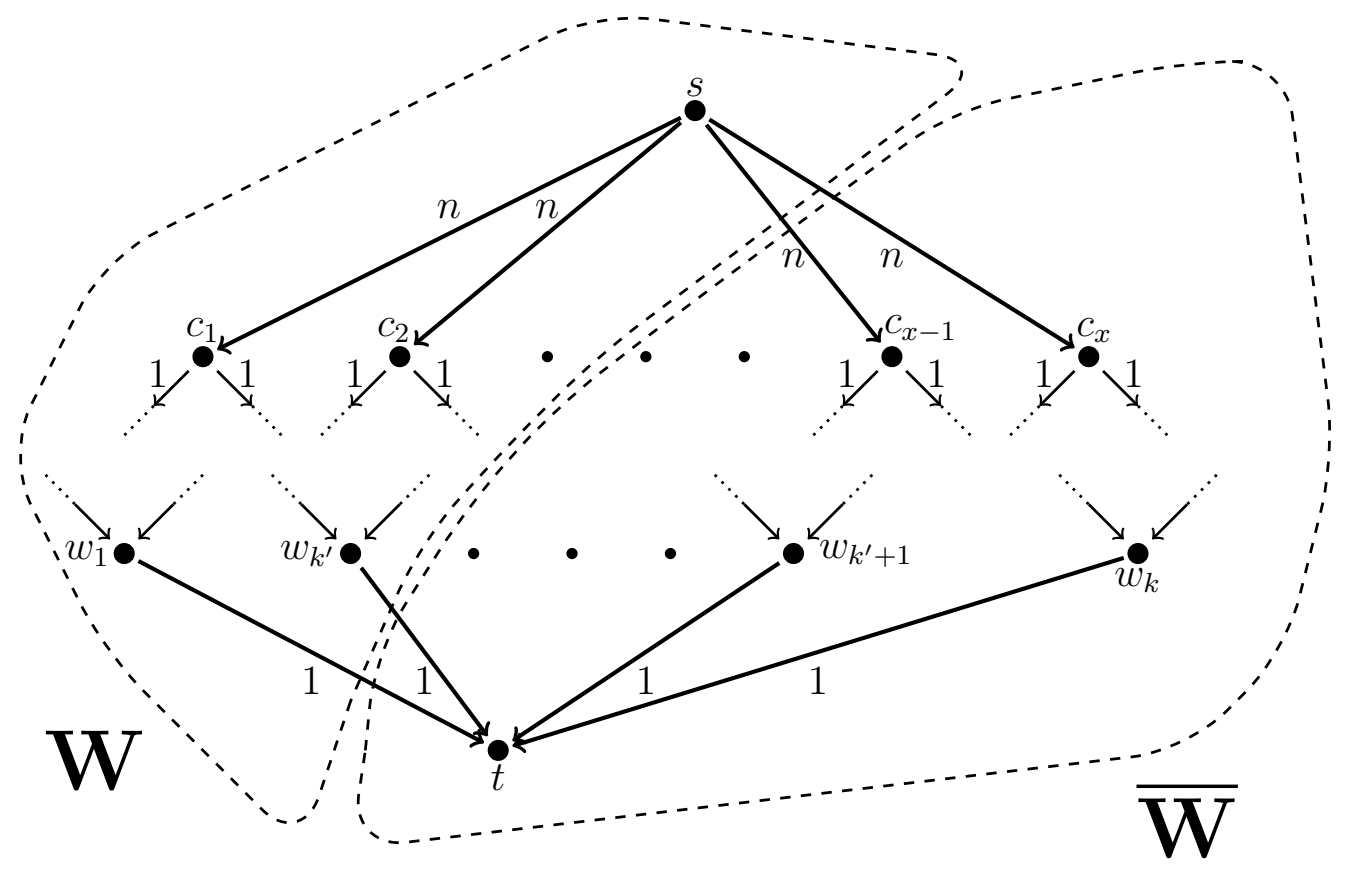

Figure 1: The Network $N$ with the $s-t$-cut $(W, \bar{W})$

- Let $V(N)=\left\{s, t, c_{1}, \ldots, c_{x}, w_{1}, \ldots, w_{k}\right\}$ where $s$ and $t$ are the source and the target of $N$ respectively, $\left\{c_{1}, \ldots, c_{x}\right\}$ is the set of colours $\{1,2, \ldots, x\}$ and $\left\{w_{1}, \ldots, w_{k}\right\}$ is the set of $k$ edges incident to the vertex $v$. (If $v$ is of degree less than $k$, we can first add edges, even multiple edges, and work with $v$ as if it is of degree $k$.)

- Add $x$ arcs, $\left[s, c_{i}\right], i=1,2, \ldots, x$, each with capacity $n$.

- Add an arc $\left[c_{i}, w_{j}\right]$ with capacity 1 for each $i=1,2, \ldots, x$ and each $j=1,2, \ldots, k$ for which the colour $c_{i}$ is admissible at $w_{j}$. 
- Add $k$ arcs, $\left[w_{j}, t\right], j=1,2, \ldots, k$, each with capacity 1 .

One can easily see that such a network has non-zero integer flow (since the capacities of all the arcs are integers). According to the Ford-Fulkerson theorem ([5]) the maximum $s-t$-flow in the network is equal to the capacity of a minimum $s-t$-cut. We shall now prove that a minimum $s-t$-cut has capacity $k$.

The arcs between $\left\{w_{1}, \ldots, w_{k}\right\}$ and $t$ form the $s-t$-cut $(T, \bar{T})$ with $T=$ $\left\{s, c_{1}, \ldots, c_{x}, w_{1}, \ldots, w_{k}\right\}$ and has capacity is $k$; hence a minimum $s-t$-cut has capacity at most $k$. On the other hand

- the cut $(S, \bar{S})$ with $S=\{s\}$ formed by all the arcs from $s$ to $\left\{c_{1}, \ldots, c_{x}\right\}$ has capacity $x \cdot n=\max \left\{\left\lceil\frac{k+1}{m+1}\right\rceil,\left\lceil\frac{k}{n}\right\rceil+2\right\} \cdot n \geq k+2 n>k$.

- the $s-t$-cut $(U, \bar{U})$ formed by all arcs from $\left\{c_{1}, \ldots, c_{x}\right\}$ to $\left\{w_{1}, \ldots, w_{k}\right\}$ has total capacity at least $k \cdot(x-2) \geq k$ since $x \geq 3$.

- any $s-t$-cut $(W, \bar{W})$, as depicted in Figure 1 , which does not contain all the arcs of either of the above three has to miss some arcs of each of the forms $\left[s, c_{i}\right],\left[c_{i}, w_{j}\right]$ and $\left[w_{j}, t\right]$; suppose it contains only $k^{\prime}<k$ of the third kind; suppose (without loss of generality) they are $w_{1}, \ldots, w_{k^{\prime}}$. If there is for each $w_{j}, j=k^{\prime}, \ldots, k$ an admissible colour in $W$, then the arcs from these admissible colours to these $w_{j}$ 's contribute at least $k-k^{\prime}$ to the capacity of this cut. Hence, in this case, the total capacity of this cut is at least $k^{\prime}+k-k^{\prime}=k$. If, on the other hand, there is a $w_{j}$ for some $j=k^{\prime}, \ldots, k$ for which there is no admissible colour in $W$, then all the (at least) $x-2$ admissible colours for this $w_{j}$ are in $\bar{W}$. But then each arc of the form $\left[s, c_{i}\right]$, for each such colour $c_{i}$, is 
then in $(W, \bar{W})$ and therefore contributes $n$ to the capacity of the cut. The total capacity of this cut is therefore at least $(x-2) \cdot n \geq k$ since $x \geq\left\lceil\frac{k}{n}\right\rceil+2$.

Now we assign the arc corresponding to $w_{j}, i=1,2, \ldots, k$ the colour $i$ such that $\left[c_{i}, w_{j}\right]$ has flow of size 1 . Since the flow is integral and the capacity of each $\operatorname{arc}\left[w_{j}, t\right]$ is 1 , the assignment is unique. The structure of the arc between $c_{i}$ and $w_{j}$ guarantees that the incidence condition is not violated and the structure and capacities of arcs originating in $s$ ensures that no colour is assigned more than $n$ times.

This completes the induction step and the proof of the theorem.

In Theorem 1, we have an upper bound for $\chi_{\mathcal{D}_{m}, \mathcal{D}_{n}}^{\prime \prime}(G)$ if $G \in \mathcal{D}_{k}$. Next we focus on the complete graph $K_{k+1}$, which is in $\mathcal{D}_{k}$ too, and find a lower bound for $\chi_{\mathcal{D}_{m}, \mathcal{D}_{n}}^{\prime \prime}(G)$. As it turns out, we only need to change the part of the maximum in the formula for $x$ which is closely related to the number of edges involved.

Theorem 2. For every three positive integers $m, n$ and $k$ we have the following $\chi_{\mathcal{D}_{m}, \mathcal{D}_{n}}^{\prime \prime}\left(K_{k+1}\right)>\max \left\{\left\lceil\frac{k+1}{m+1}\right\rceil,\left\lceil\frac{k(k+1)}{n+2 k n-n^{2}}\right\rceil\right\}-1$.

Proof. In this proof, we denote, for every three positive integers $m, n$ and $k$, the value $\max \left\{\left\lceil\frac{k+1}{m+1}\right\rceil,\left\lceil\frac{k(k+1)}{n+2 k n-n^{2}}\right\rceil\right\}$ by $y$. Suppose, for a proof by contradiction, that $\chi_{\mathcal{D}_{m}, \mathcal{D}_{n}}^{\prime \prime}\left(K_{k+1}\right) \leq y-1$ and consider any total $\left(\mathcal{D}_{m}, \mathcal{D}_{n}\right)$-colouring of $K_{k+1}$ using only $y-1$ colours. Then, since $y=\max \left\{\left\lceil\frac{k+1}{m+1}\right\rceil,\left\lceil\frac{k(k+1)}{n+2 k n-n^{2}}\right\rceil\right\}$, we have that $y-1<\left\lceil\frac{k+1}{m+1}\right\rceil$ or $y-1<\left\lceil\frac{k(k+1)}{n+2 k n-n^{2}}\right\rceil$ and hence that $y-1<\frac{k+1}{m+1}$ or $y-1<\frac{k(k+1)}{n+2 k n-n^{2}}$. 
In order to show that such a total $\left(\mathcal{D}_{m}, \mathcal{D}_{n}\right)$-colouring of $K_{k+1}$ does not exist, we consider two cases:

Case 1: $y-1<\frac{k+1}{m+1}$

In this case we have that $(y-1)(m+1)<k+1$. Hence, no matter how we colour the vertices of $K_{k+1}$ using $y-1$ colours with at most $m+1$ vertices receiving the same colour (which is the best we can do since any monochromatic set with more than $m+1$ vertices induces a complete subgraph which is regular of degree more than $m+1$ and hence is not in $\mathcal{D}_{m}$ ), not all of the $k+1$ vertices of $K_{k+1}$ will be coloured.

Case 2: $y-1<\frac{k(k+1)}{n+2 k n-n^{2}}$

In this case we have $(y-1)\left(n+2 k n-n^{2}\right)<k(k+1)$ and we shall show that it is impossible to colour the edges of $K_{k+1}$ in $y-1$ colours of which each monochromatic set of edges induces a subgraph of $K_{k+1}$ which is in $\mathcal{D}_{n}$.

Suppose, to the contrary, that there is such a colouring of the edges of $K_{k+1}$. Consider a labelling $v_{1}, \ldots, v_{k+1}$ of the vertices of $K_{k+1}$ and a labelling $c_{1}, \ldots, c_{y-1}$ of the colours used. One can clearly change the colours of (some) edges of colour $c_{i}$ with $i>1$ to have colour $c_{1}$ (if needed) and choose another labelling of the vertices (if needed) until the subgraph induced by the set of edges of colour $c_{1}$ consists of:

- a complete graph on the vertices $v_{1}, \ldots, v_{n+1}$, while

- each of the $k-n$ vertices $v_{i}$ with $i>n+1$ is adjacent to exactly $n$ vertices with smaller labels.

The subgraph induced by the set of edges of $K_{k+1}$ of colour $c_{1}$ then has exactly $\frac{n(n+1)}{2}+(k-n) n$ edges while the subgraph induced by the set of 
edges of $K_{k+1}$ of every other colour $c_{i}$ then has at most this number of edges. But then the total number of edges which are coloured is at most

$$
(y-1)\left(\frac{n(n+1)}{2}+(k-n) n\right)=\frac{(y-1)\left(n+2 k n-n^{2}\right)}{2}<\frac{k(k+1)}{2},
$$

by the assumption of this case. This, however, is a contradiction since $K_{k+1}$ has $\frac{k(k+1)}{2}$ edges.

This completes the proof of the theorem.

It is not very difficult to see that the expression $\frac{k}{n}+2$ majorizes the expression $\frac{k(k+1)}{n+2 k n-n^{2}}+1$ if $k \geq n-1$. Furthermore, $\frac{k+1}{m+1}$ majorizes $\frac{k}{n}+2$ if $k(n-m-1) \geq n(2 m+1)$. Hence the value of the invariant is often equal to $\left\lceil\frac{k+1}{m+1}\right\rceil$.

\section{Acknowledgement}

The first author is thankful to the P.J. Šafárik University, Košice, Slovakia whose hospitality he enjoyed during the preparation of this paper; he is also supported in part by the National Research Foundation of South Africa (Grant Numbers 90841, 91128).

The research of the second author was also supported under the grant numbers APVV-15-0091 and VEGA 1/0142/15 and projects ITMS 26220120007 and ITMS 26220220182.

\section{References}

[1] M. Borowiecki, I. Broere, M. Frick, P. Mihók, G. Semanišin, Survey of hereditary properties of graphs, Discuss. Math. Graph Theory 17 (1997) 5-50. doi:10.7151/dmgt.1037. 
[2] M. Borowiecki, A. Kemnitz, M. Marangio, P. Mihók, Generalized total colorings of graphs, Discuss. Math. Graph Theory 31 (2011) 209-222. doi:10.7151/dmgt.1540.

[3] W. Dargie, C. Poellabauer, Fundamentals of wireless sensor networks: theory and practice, Wiley, 2010. doi:10.1002/9780470666388.

[4] R. Diestel, Graph Theory, Springer, Budapest, 1997.

[5] L. Ford, D. Fulkerson, Maximal flow through a network, Canad. J. Math. 8 (1956) 399-404. doi:10.4153/CJM-1956-045-5.

[6] F. Galčík, G. Semanišin, Centralized broadcasting in radio networks with k-degenerate reachability graphs, in: ITAT 2006 Information Technologies - Applications and Theory, Bystrá dolina, Slovakia, 2006, pp. 41-46.

[7] S. Isobe, X. Zhou, T. Nishizeki, Total Colorings Of Degenerate Graphs, Combinatorica 27 (2007) 167-182. doi:10.1007/s00493-007-0050-5.

[8] R. Lick, A. T. White, k-degenerate graphs, Canad. J. Math. 22 (1970) 1082-1096. doi:10.4153/CJM-1970-125-1.

[9] P. Mihók, Minimal reducible bounds for the class of $k$-degenerate graphs, Discrete Math. 236 (2001) 273-279. doi:10.1016/S0012-365X(00)00447-7.

[10] G. Semanišin, Minimal reducible bounds for induced-hereditary properties, Discrete Math. 286 (2004) 163-170. doi:10.1016/j.disc.2003.11.058. 